\title{
RETRACTED ARTICLE: Wearable loT based cloud assisted framework for swimming persons in health monitoring system
}

\author{
Hongyan Zhou ${ }^{1}$. Carlos Enrique Montenegro-Marin ${ }^{2} \cdot$ Ching-Hsien $\mathrm{Hsu}^{3}$ \\ Published online: 14 June 2020 \\ (C) Springer Science+Business Media, LLC, part of Springer Nature 2020
}

The Editor-in-Chief has retracted this article "Wearable IoT based cloud assisted framework for swimming persons in health monitoring systems". The article was accepted as part of a guest-edited special issue in Current Psychology. Before the special issue was finalized, the Editor-in-Chief detected problems with editorial handling and peer review and decided not to proceed with the special issue. Post publication peer review found the following issues:

- The authors claim to have carried out a thorough review of the literature, but there is no information on how papers were identified to be included as part of the background study.

- The literature review is largely incomprehensible and incomplete; the lack of a comprehensive and clear description about existing systems and the drawbacks with them means that the manuscript lacks a rationale for the authors' proposed "Cloud Assisted Recurrent Neural Network" system.

- The Results and Discussion section suggests the authors applied a neural network with data from swimmers, but

Hongyan Zhou

359370154@qq.com

Carlos Enrique Montenegro-Marin cemontenegrom@udistrital.edu.co

Ching-Hsien Hsu

robertchh@asia.edu.tw

1 School of Physical Education, Hunan University of Arts and Science, Changde 415000, Hunan, China

2 Facultad de ingeniería, Universidad Distrital Francisco José de Caldas, Bogotá, Colombia

3 Asia University, Taichung City, Taiwan there is no information about how these data (in Table 1 and Table 2) were collected, or where the sensors were worn. This prevents evaluation of the effectiveness of the neural network that has been developed.

- In the Outcomes Discussed section, the authors state that the swimming mechanism is easily captured by the movement of trunk and limb action. As there is no information about where the sensors are placed or how the data is captured, there does not appear to be support for this statement in the paper.

The Editor-in-Chief therefore no longer has confidence in the reliability of the work presented.

Dr. Ching-Hsien Hsu does not agree to this retraction. Dr. Hongyan Zhou and Dr. Carlos Enrique Montenegro-Marin did not respond to any correspondence from the Editor about this retraction.

The online version of this article contains the full text of the retracted article as Supplementary Information.

Supplementary Information The online version contains supplementary material available at https://doi.org/10.1007/s12144-020-00822-0. 